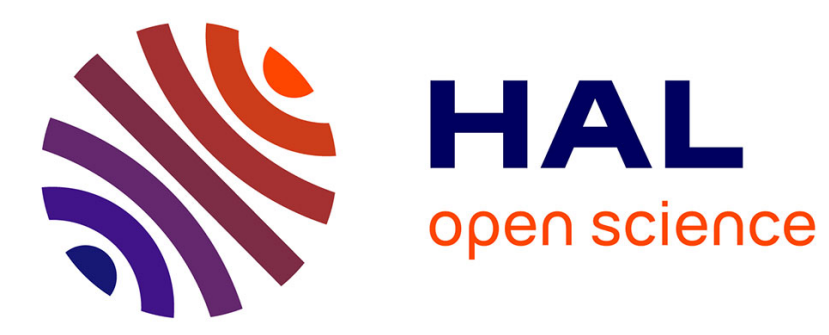

\title{
A parallel multiple reference point approach for multi-objective optimization
}

José Rui Figueira, Arnaud Liefooghe, El-Ghazali Talbi, Andrzej P. Wierzbicki

\section{To cite this version:}

José Rui Figueira, Arnaud Liefooghe, El-Ghazali Talbi, Andrzej P. Wierzbicki. A parallel multiple reference point approach for multi-objective optimization. European Journal of Operational Research, 2010, 205 (2), pp.390 - 400. 10.1016/j.ejor.2009.12.027 . hal-00522619

\section{HAL Id: hal-00522619 \\ https://hal.science/hal-00522619}

Submitted on 4 Oct 2010

HAL is a multi-disciplinary open access archive for the deposit and dissemination of scientific research documents, whether they are published or not. The documents may come from teaching and research institutions in France or abroad, or from public or private research centers.
L'archive ouverte pluridisciplinaire HAL, est destinée au dépôt et à la diffusion de documents scientifiques de niveau recherche, publiés ou non, émanant des établissements d'enseignement et de recherche français ou étrangers, des laboratoires publics ou privés. 


\title{
A Parallel Multiple Reference Point Approach for Multi-objective Optimization
}

\author{
J. R. Figueira ${ }^{\mathrm{a}, \mathrm{b}}$, A. Liefooghe ${ }^{\mathrm{c}, \mathrm{d}}$, E.-G. Talbi ${ }^{\mathrm{c}, \mathrm{d}}$, A. P. Wierzbicki ${ }^{\mathrm{e}}$ \\ ${ }^{a}$ CEG-IST, Center for Management Studies, Instituto Superior Técnico, Technical \\ University of Lisbon, Tagus Park, Av. Cavaco Silva, 2780-990 Porto Salvo, Portugal \\ ${ }^{b}$ Associate Researcher at LAMSADE, UMR CNRS 7024, Université Paris-Dauphine, \\ Place du Maréchal De Lattre de Tassigny, 75775 Paris Cedex, France \\ ${ }^{c}$ LIFL, UMR CNRS 8022, Université Lille 1, \\ Bât. M3, 59655 Villeneuve d'Ascq Cedex, France \\ ${ }^{d}$ INRIA Lille-Nord Europe, \\ Parc Scientifique de la Haute Borne, 40 av. Halley, 59650 Villeneuve d'Ascq, France \\ ${ }^{e}$ National Institute of Telecommunications, Szachowa Str. 1, 04-894 Warsaw, Poland
}

\begin{abstract}
This paper presents a multiple reference point approach for multi-objective optimization problems of discrete and combinatorial nature. When approximating the Pareto Frontier, multiple reference points can be used instead of traditional techniques. These multiple reference points can easily be implemented in a parallel algorithmic framework. The reference points can be uniformly distributed within a region that covers the Pareto Frontier. An evolutionary algorithm is based on an achievement scalarizing function that does not impose any restrictions with respect to the location of the reference points in the objective space. Computational experiments are performed on a bi-objective flow-shop scheduling problem. Results, quality measures as well as a statistical analysis are reported in the paper.

Key words: multiple objective programming, parallel computing, multiple reference point approach, evolutionary computations, bi-objective flow-shop scheduling
\end{abstract}

Email addresses: figueira@ist.utl.pt (J. R. Figueira), arnaud.liefooghe@lifl.fr (A. Liefooghe), talbi@lifl.fr (E.-G. Talbi), a.wierzbicki@itl.waw.pl (A. P. Wierzbicki) 


\section{Introduction}

Multi-objective Optimization (MO) is one of most challenging areas in the field of Multiple Criteria Decision Analysis (MCDA). Over the last decades, a large number of papers were published in this field comprising both theoretical and applied works. The most challenging problems in $\mathrm{MO}$ are related to the identification of the Pareto Frontier $(\mathrm{PF})$, or an approximation of it $\left(\mathrm{PF}^{A}\right)$ for large-size and rather difficult $\mathrm{MO}$ problems. For such a purpose, evolutionary algorithms seem more adequate than exact methods. However, the identification of the whole set or of an approximation of the PF is frequently not necessary, an approximation of some specific regions suffices. Indeed, when some preference information is provided by the Decision-Maker (DM), diverse methods can guide, in an interactive manner, the search of the potentially best compromise solution(s) (which is an efficient solution) in a particular region of interest. Reference point methods are particularly adequate to deal with this kind of situations; the preference information needed by them has mainly the form of reference point(s) (or also any other information that can be translated into reference point(s)). Reference point-based methods use then an achievement scalarizing function to make projection of the reference points onto the PF.

Contrary to the single-objective case, typically there is no unique optimal solution for a MO problem. Instead, a set of solutions, called Pareto solutions, efficient solutions, etc, represent the PF when transformed into the objective space. A fundamental issue while trying to solve MO problems is related to the cooperation between the DM and a computerized Decision Support System (DSS). In general, the DSS includes a mathematical model of the problem being solved along with a data base, an optimization solver, and an interactive solution procedure. There are several approaches to the roles that the DM could play in a decision-making process. Firstly, the a priori approaches, where the DM is supposed to provide some knowledge or preferences about the problem to be solved in order to help the DSS in its search; practical experience shows that such methods are seldom effective. Separately, the a posteriori approaches, where the DSS aims at finding, or approximating the whole set of efficient solutions; the DM then has to choose his/her most preferred one. Finally, in interactive approaches, there is a progressive direct interaction/cooperation between the DM and the DSS.

Over the last two decades, most of MO resolution methods proposed in the literature were rather the a posteriori ones. A large part of them consist 
of approximating the set of efficient solutions and the corresponding PF using an evolutionary algorithm. On the one hand, this is based on the belief that the computing power of modern computers is unlimited, we can use them for any complex problems and solution methods. This belief, however, is contradicted by computational experience of solving complex problems: even the most powerful computer of any generation can be easily saturated, due to the non-linear dependencies of computational complexity on the amount of data processed. Thus, a reasonable use of the existing computing power, even if this power is tremendous due to the possibilities of parallel use of computers in the network, still remains and will probably always remain a fundamental problem. On the other hand, many interactive approaches are based on the reference point method using achievement scalarizing functions as proposed by Wierzbicki (1980) and developed by many other researchers; see, for example, Wierzbicki et al. (2000). The reference point method results in projecting a given reference point (or a pair of them, usually called reservation and aspiration points), that represents the objective, criteria or outcome values desired by the DM, onto the set of efficient solutions. There are diverse interaction protocols within the framework of reference point approaches, starting with just fully sovereign change of reference points by the DM, through using additional trade-off information, up to visual interfaces based on fuzzy specification of reference values. However, the result is focusing on a specific region of the objective space, thus avoiding the loss of computational resources for searching solutions that may not interest the DM at the end.

In this paper, we propose a new method combining the use of reference points while trying to approximate the whole set of efficient solutions, or a selected part of it. Instead of using a single reference point, the idea of this a posteriori approach is to automatically define a set of points in such a way that the objective space is uniformly divided, but entirely covered. Each point gives rise to a corresponding achievement scalarizing function that concentrates on a specific sub-region of the objective space. Thus, the set of efficient solutions can be rebuilt by combining the output of all solvers. Notice that the solvers can be launched in parallel since the problems of optimizing a given achievement function can all be solved independently. The proposed parallel multiple reference point approach can be used to solve difficult real-world optimization problems, and it is here applied to a biobjective combinatorial scheduling problem.

The outline of the paper is as follows. Section 2 introduces some funda- 
mental concepts related to MO. Section 3 is devoted to the multiple reference point approach proposed in the paper; the key issues being widely detailed. Section 4 presents the parallel model and the implementation of the method. Section 5 formulates a bi-objective Flowshop Scheduling Problem (FSP). Section 6 shows the effectiveness of our approach by conducting experiments on the FSP. Finally, the last section concludes the paper and draws some perspectives.

\section{Multi-objective Optimization (MO)}

Many areas of the industry as, for example, telecommunications, transportation, aeronautics, chemistry, mechanical, and environment, deal with MO, where various conflicting objectives have to be considered simultaneously. This section briefly presents some basic concepts, definitions, and notation for MO. The interested reader is referred to Miettinen (1999); Deb (2001); Coello Coello et al. (2002) for more details about this field.

\subsection{Basic Concepts}

A general MO problem consists of optimizing a set of $n \geq 2$ objective functions $f_{1}(x), f_{2}(x), \ldots, f_{n}(x)$. Each objective function can be either minimized or maximized; or even stabilized, kept close to a given target level (Wierzbicki et al., 2000). Here we assume, without loss of generality, that all are to be minimized. A decision vector $x=\left(x_{1}, x_{2}, \ldots, x_{k}\right)$ is represented by a vector of $k$ decision variables. Let $X$ denote the set of feasible solutions in the decision space $\mathbb{R}_{0}^{k}\left(X \subseteq \mathbb{R}_{0}^{k}\right)$. To each decision vector $x \in X$ is assigned exactly one objective vector, $z \in Z$, on the basis of a vector function $f: X \rightarrow Z$ with $z=\left(z_{1}, z_{2}, \ldots, z_{n}\right)=f(x)=\left(f_{1}(x), f_{2}(x), \ldots, f_{n}(x)\right)$, where $Z=f(X)$ denotes the set of feasible points in the objective (or criterion) space $\mathbb{R}^{n}$ $\left(Z \subseteq \mathbb{R}^{n}\right)$. Therefore, a MO problem can be formulated as follows:

$$
\begin{aligned}
\text { "min" } & f(x)=\left(f_{1}(x), f_{2}(x), \ldots, f_{n}(x)\right) \\
\text { subject to: } & x \in X
\end{aligned}
$$

Whereas solving a single-objective optimization problem generally results in a unique optimal solution, a MO problem obtains rather a set of solutions known as Pareto optimal. A fundamental concept is the one of dominance that can be defined as follows. 
Definition 1 (Dominance). A solution $x_{1} \in X$ dominates another solution $x_{2} \in X$ if and only if $\forall i \in\{1, \ldots, n\}, f_{i}\left(x_{1}\right) \leq f_{i}\left(x_{2}\right)$ and $\exists j \in$ $\{1, \ldots, n\}$ such that $f_{j}\left(x_{1}\right)<f_{j}\left(x_{2}\right)$.

The following two concepts depend on the dominance concept.

Definition 2 (Efficiency). A solution $x^{*} \in X$ is efficient if and only if there is not another solution $x \in X$ such that $x$ dominates $x^{*}$.

The whole set of efficient solutions is the Pareto optimal set, and is denoted by $X_{P}$. The image of a Pareto optimal solution in the objective space results in a non-dominated outcome vector.

Definition 3 (Non-dominated outcome vector). A point $z \in Z$ is a non-dominated outcome vector if there exists at least one efficient solution $x \in X_{P}$ such that $z=f(x)$.

The set of all non-dominated outcome vectors is the Pareto Frontier (PF). One of the possible approaches for solving $\mathrm{MO}$ problems consists of finding $\mathrm{PF}$ or an approximation $\mathrm{PF}^{A}$. This depends on the practical computational complexity of the problem, because finding a representation of $\mathrm{PF}$ is practically possible only if the resulting computational complexity is rather low.

Now, suppose that the optimum is known for each objective function, then we can define the concept of ideal vector:

Definition 4 (Ideal vector). The ideal vector $z^{*}=\left(z_{1}^{*}, z_{2}^{*}, \ldots, z_{n}^{*}\right)$ is the vector that optimizes each objective function individually

$$
z_{i}^{*}=\min _{x \in X} f_{i}(x)
$$

Of course, this ideal vector optimizing each objective function is rarely feasible as the objectives are often in conflict. Besides, the upper bounds for all objectives of the PF can be represented by the nadir point $z^{n}$. This nadir point is much more difficult or impossible to compute (Miettinen, 1999), especially when the number of objectives is more than two. A rough approximation of the nadir point can be provided by recording the maximal values of all objective functions obtained from their separate minimization, while determining the ideal point. 


\subsection{Achievement Scalarizing Functions}

The achievement scalarizing function approach, proposed by Wierzbicki (1980), is frequently used to solve MO problems. This technique is particularly well suited to work with reference points. A reference point gives desirable or acceptable values for each one of the objective functions. These objective values are called aspiration levels and the resulting objective vector is called a reference point and can be defined either in the feasible or in the infeasible region of the objective space. One of the families of achievement functions can be stated as follows:

$$
\sigma\left(z, z^{0}, \lambda, \rho\right)=\max _{i=1,2, \ldots, n}\left\{\lambda_{i}\left(z_{i}-z_{i}^{0}\right)\right\}+\rho \sum_{i=1}^{n} \lambda_{i}\left(z_{i}-z_{i}^{0}\right)
$$

where $\sigma$ is a mapping from $Z$ onto $\mathbb{R}, z=\left(z_{1}, z_{2}, \ldots, z_{n}\right)$ is an objective vector, $z^{0}=\left(z_{1}^{0}, z_{2}^{0}, \ldots, z_{n}^{0}\right)$ is a reference point vector, $\lambda=\left(\lambda_{1}, \lambda_{2}, \ldots, \lambda_{n}\right)$ is a scaling coefficients vector (weighting coefficients), and $\rho$ is an arbitrary small positive number $(0<\rho \ll 1)$. The word family is used here to state that several functions can be built according to the variability of the weighting coefficients and the reference points.

Now, the following achievement problem can be built:

$$
\begin{aligned}
\min & \sigma\left(z, z^{0}, \lambda, \rho\right) \\
\text { subject to: } & x \in X
\end{aligned}
$$

For a given reference point $z^{0}$, two properties can be proved (Steuer, 1986):

i) if $x^{\star}=\arg \min _{x \in X} \sigma\left(z, z^{0}, \lambda, \rho\right)$, then $x^{\star}$ is an efficient solution;

ii) if $x^{\star}$ is an efficient solution, then there exists a function $\sigma\left(z, z^{0}, \lambda, \rho\right)$ such that $x^{\star}$ is a (global) optimal solution of the achievement problem given in (3).

Note that the weighting vectors can be normalized, and the set of all feasible normalized weighting vectors can be represented as follows:

$$
\Lambda=\left\{\lambda=\left(\lambda_{1}, \lambda_{2}, \ldots, \lambda_{n}\right) \mid \sum_{i=1}^{n} \lambda_{i}=1, \quad \lambda_{i}>0, i=1,2, \ldots, n\right\}
$$

Using different $\lambda$ vectors for the same reference point $z^{0}$ can lead to different optimal solutions for the achievement problem defined in (3). This aspect can be used to design a parallel algorithm dealing with the same reference point and different weighting vectors. 


\section{A Multiple Reference Point Approach}

The goal of the proposed approach in this paper is to approximate the whole PF by using multiple reference points. Using several reference points is particularly well-suited to parallel computing since the resulting problems can all be solved independently. The general principle of the algorithm consists of generating a given number of reference points. These points are generated according to, either a rough approximation of the bounds of the $\mathrm{PF}^{A}$, or by reasonable upper and lower bounds provided by the DM. For each reference point, a solver is assigned to it. This solver aims at finding or approximating the efficient set corresponding to the pre-defined reference point (and some weighting coefficients). The reference point solvers are all launched in a parallel way and a master process preserves and then merges the approximated efficient set found by these subprocesses.

In this section, the multiple reference point approach is described after having introduced some essential issues relating to its design.

\subsection{Fundamental Issues}

This subsection introduces some fundamental aspects for the design of the multiple reference point method. Firstly, a way of estimating the bounds of the $\mathrm{PF}^{A}$ is given. Secondly, a description of how to generate multiple reference points is detailed. Finally, a single reference point solver is proposed to be used as a subroutine of the main algorithm.

\subsubsection{Estimating the Bounds of the Pareto Frontier}

As pointed out in Subsection 2.1, computing the nadir point $z^{n}$ is rather a hard task, and even more difficult for discrete MO problems of large-size. Furthermore, computing the exact ideal point $z^{*}$ is usually unfeasible as soon as we deal with large-size instances. However, in the work established in this paper, they are required to generate the initial set of reference points to be used by the algorithm. We should then find only a sufficiently good approximation of these two points. These approximations will be denoted by $z^{*^{A}}$ and $z^{n^{A}}$, respectively.

Let us consider two legitimate options for performing such a task. On the one hand, if the DM is able to provide reasonable upper and lower bounds for each objective function, ideal and nadir computations or approximations rely then upon such an initial scaling information. From our experience, it is always conceivable to ask the DM to give some pieces of initial preference 
information or knowledge about the problem to be solved. Furthermore, let us notice that metaheuristic algorithms can, in general, not run fully automatically without some initial information. At least, for instance, the starting solution or population, even if provided by some random number generator, usually needs some initial scaling.

On the other hand, if the DM is not able to provide any piece of preference information, another option is to approximate these points by means of a related search method to be performed over a relatively small number of iterations. Generally speaking, evolutionary multi-objective algorithms give set-valued but discrete approximations of the $\mathrm{PF}^{A}$; the ideal and the nadir points of such approximations can then be computed. The difficulty of estimating nadir points is related to the original problem and to the fact that such evolutionary approximations might badly approximate a PF close to the nadir point. Therefore, it is important to apply at least two aspects of an evolutionary multi-objective algorithm. One is to use a strategy stressing diversity, or generally enhancing the importance of the extreme values of the discrete set-valued approximation of the PF; there might be several such strategies (Szczepański and Wierzbicki, 2003). Another is to use a stopping criterion that involves the diameter of the approximation of the $\mathrm{PF}$, measured as the distance between the nadir and the ideal points. If this diameter stabilizes in subsequent iterations, it indicates that either the approximation is already good (because a good approximation of the nadir point is one of the most difficult aspects of the entire $\mathrm{PF}^{A}$ ) or that the algorithm specifically applied cannot produce further improvement (which can always happen with heuristic algorithms). Szczepański and Wierzbicki (2003) provide a good overview of diverse examples. Notice that the approximation must be corrected by broadening it by some factor. This "broadening" is necessary for many reasons, starting with the fact that an evolutionary multi-objective algorithm approximates the distance ideal-nadir from below, and such "broadening" is even used when running classical nonlinear multiobjective optimization.

To approximate the ideal and the nadir points, Deb et al. (2006) proposed a modified NSGA-II procedure that focuses its search towards the extremities of the PF. Then, the best and worst objective values are computed among the final $\mathrm{PF}^{A}$, and can be used to constitute the approximated ideal and nadir objective vectors, $z^{*^{A}}$ and $z^{n^{A}}$. To do so, this algorithm uses a diversity maintaining strategy, based on an extremized crowding distance that emphasizes the importance of the worst objective solutions. The reader is 


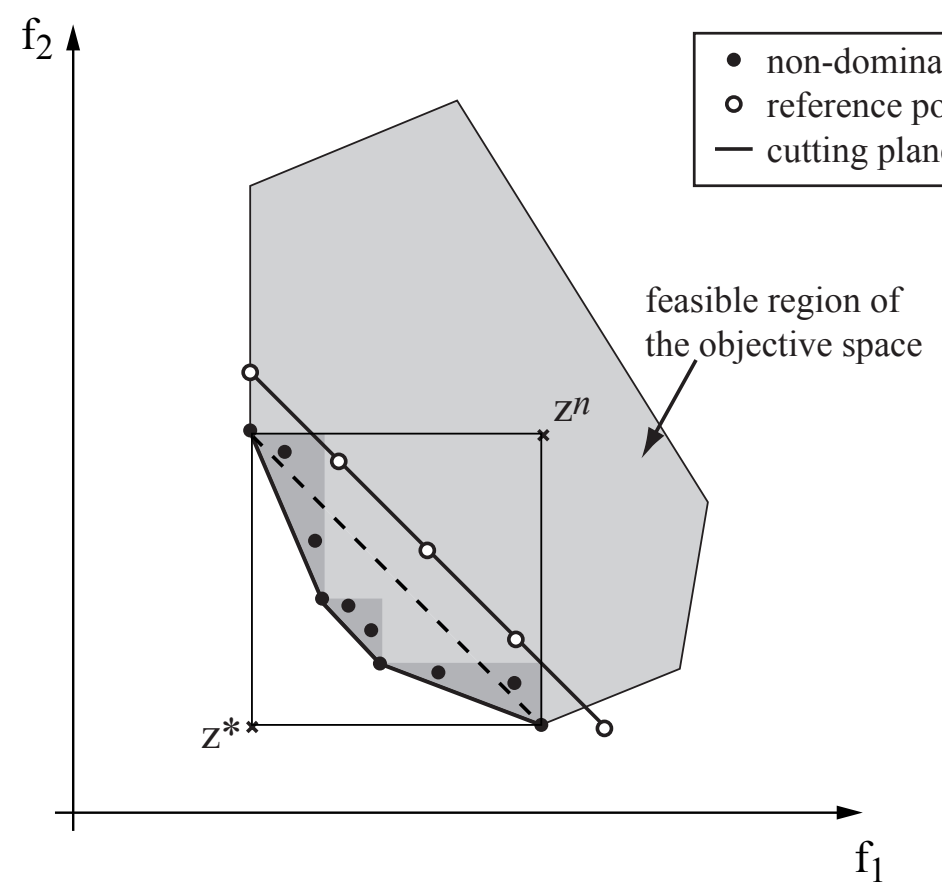

Figure 1: Cutting plane in the objective space for a two-objective problem.

referred to Deb et al. (2006) for more details about this approach.

\subsubsection{Generating Multiple Reference Points}

There might exist several ways of generating multiple reference points. The most intuitive is perhaps the random generation over a predefined area of the objective space. This simple way, however, does not seem to be adequate to our case. The random generation can be so random that it does not cover a predefined area. This is the reason why we propose to define a quite uniform distribution of the multiple points over a predefined area. After having defined the bounds for each objective function, an area is built in such a way that it covers all the feasible region of the objective space. Figure 1 shows how to proceed when two objectives are involved. Let us assume, for the sake of simplicity, that the bounds can be defined in a more or less exact way. How to proceed? A possible way is to perform the following steps:

1. Compute the ideal point $z^{*}$ or a good approximation of it $z^{*^{A}}$;

2. Compute the nadir point $z^{n}$ or a good approximation of it $z^{n^{A}}$; 
3. Consider the box formed by these points, and define a cutting plane as the "diagonal" of this box;

4. If necessary, relax the extreme points of such a cutting plane and obtain the cutting plane used to generate the reference points;

5. Uniformly generate the reference points, according to the number of processors available.

Figure 1 illustrates how to define this cutting plane. There are two embedded rectangles. The small one was built from $z^{*}$ and $z^{n}$. The diagonal, represented by the dashed line connecting the two opposing vertices can be used as a first cutting plane. Then, we relax this line in order to get the solid style one. This is done to avoid the use of the extreme points as reference ones, since they are non-dominated. Obviously, when dealing with approximations of the ideal and nadir points, we do not need to perform such a relaxation. In continuous multi-objective linear models, the plane covers the whole non-dominated region which is above the plane (when all the objective functions are to be minimized). In discrete MO, it is, however, not always the case as we can observe in Figure 1 where we just build a cutting plane by moving a little bit up the dashed line. There are non-dominated outcome points below and above the cutting line. This does not raise any problem since the achievement function that will be used does not impose any constraints on the location of the reference points. Such a conclusion is not true when using a (weighting) Chebychev metric, as it is the case in many research works done in interactive decision-making tools.

The following figure shows how to define initial reference points for a three objectives problem. In such a case, the area is represented by a triangle. It shows a grid of fifteen reference points, built from the ideal point $z^{*}$ and the nadir point $z^{n}$, or good approximation of them $\left(z^{*^{A}}\right.$ and $z^{n^{A}}$, respectively). Note that this principle can be easily generalized for $n$-dimensional problems, but the required number of reference points grows exponentially with $n$.

\subsubsection{A Single Reference Point Evolutionary Algorithm}

In this subsection, we present an evolutionary algorithm based on the achievement functions introduced in Subsection 2.2 and designed to be used with a single reference point $z^{0}$ and a single weighting coefficients vector $\lambda$. This single reference point search method will appear as a subroutine of the main algorithm in order to solve the problem resulting from $z^{0}$ and $\lambda$. Note that this is not a pure single-objective optimization problem since we are 


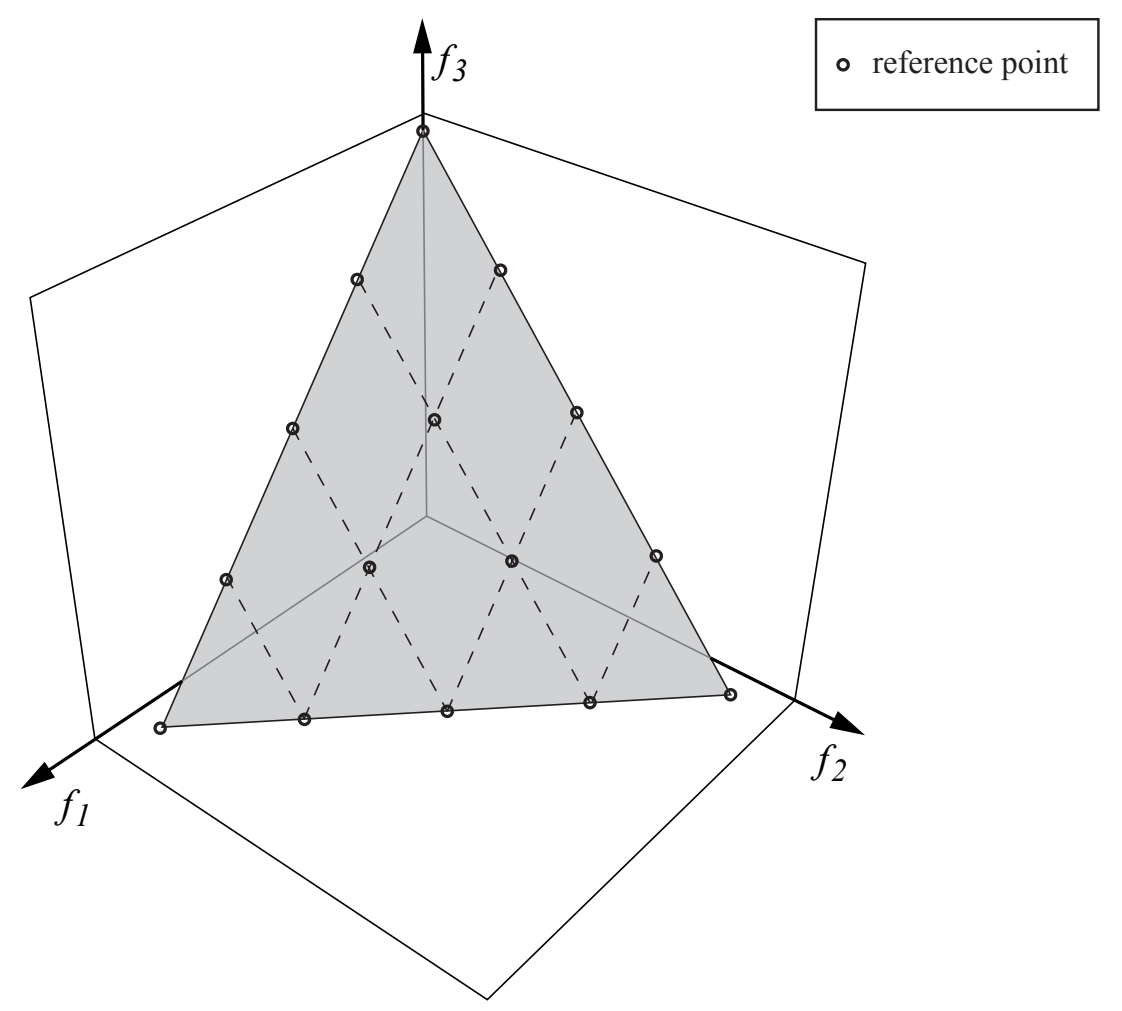

Figure 2: Generating initial reference points for a three-objective problem.

interested in getting a certain number of non-dominated solutions. This is illustrated in Figure 3 and Figure 4. Different kinds of methods can be used to tackle such a problem (exact methods, heuristics, or metaheuristics), and here we choose to design an evolutionary algorithm. It consists of the Preference-Based Evolutionary Algorithm (PBEA) proposed by Thiele et al. (2009). As the problem function is used to get efficient solutions, now we can talk in terms of approximate efficient solutions. Then, PBEA aims at producing a good, probably small, set of approximate efficient solutions related to $z^{0}$ and $\lambda$.

PBEA is a variant of the Indicator-Based Evolutionary Algorithm (IBEA) proposed by Zitzler and Künzli (2004), where preference information is taken into account through a reference point. The main idea behind IBEA is to introduce a total order between solutions by generalizing the dominance relation given in Definition 1 by means of an arbitrary binary quality indicator $I$. Indeed, its fitness assignment scheme is based on a pairwise comparison of 

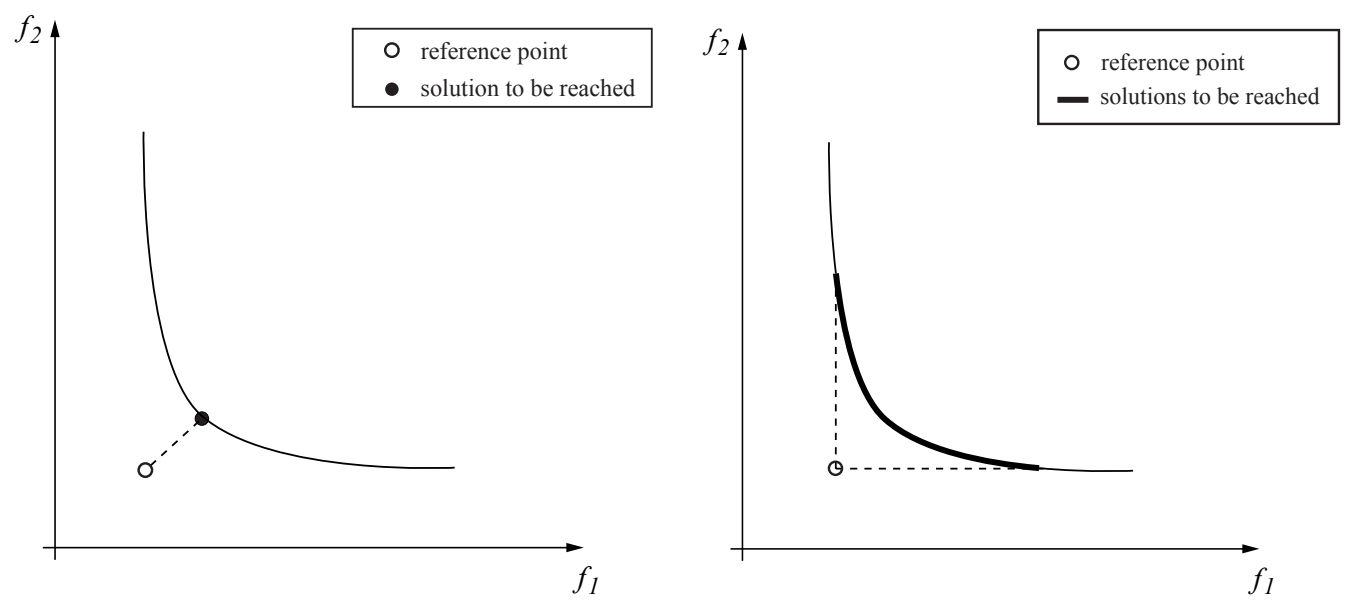

Figure 3: Traditional reference point ap- Figure 4: Multiple solutions are to be found. proach.

solutions from the current population, based on a user-given indicator $I$. To each individual $x$ is then assigned a fitness value $F(x)$ measuring the "loss in quality" if $x$ was removed from the current population (Zitzler and Künzli, 2004). Selection for reproduction consists of a binary tournament between randomly chosen individuals. And selection for replacement consists of iteratively removing the worst solution from the current population until the required population size is reached. Fitness information of the remaining individuals is updated each time there is a deletion. A detailed description of IBEA is reproduced below.

Several binary indicators can be used in (4). As an example, Zitzler and Künzli (2004) define the binary additive $\epsilon$-indicator $\left(I_{\epsilon+}\right)$ as follows:

$$
I_{\epsilon+}\left(x, x^{\prime}\right)=\max _{i \in\{1, \ldots, n\}}\left\{f_{i}(x)-f_{i}\left(x^{\prime}\right)\right\}
$$

It computes the minimum value by which a solution $x \in X$ has to be, or can be, translated in the objective space to weakly dominate another solution $x^{\prime} \in$ $X$.

In order to take preference information into account by means of a reference point, Thiele et al. (2009) propose the so-called preference-based quality indicator based on the achievement function given in Definition 2. First, a normalized achievement function is defined.

$$
\sigma\left(f(x), z^{0}, \lambda, \rho, \delta\right)=\sigma\left(f(x), z^{0}, \lambda, \rho\right)+\delta-\min _{x^{\prime} \in P}\left\{\sigma\left(f\left(x^{\prime}\right), z^{0}, \lambda, \rho\right)\right\}
$$


Indicator-Based Evolutionary Algorithm (IBEA).

1. Initialization. Start with a user-given initial population $P$ of size $N$, or generate it randomly.

2. Fitness assignment. Compute the fitness values of individuals in $P$ :

$$
F(x) \leftarrow \sum_{x^{\prime} \in P \backslash\{x\}}\left(-e^{-I\left(x^{\prime}, x\right) / \kappa}\right)
$$

where $\kappa>0$ is a user-defined scaling factor.

3. Environmental selection. Iterate the following steps until the size of the population $P$ does not exceed $N$ :

(a) Choose an individual $x^{\star} \in P$ with the smallest fitness value: $F\left(x^{\star}\right) \leq F(x)$ for all $x \in P$.

(b) Remove $x^{\star}$ from $P$.

(c) Update the fitness values of the remaining individuals. For all $x \in P$ :

$$
F(x) \leftarrow F(x)+e^{-I\left(x^{\star}, x\right) / \kappa}
$$

4. Termination. If a stopping condition is satisfied, return the nondominated solutions of $P$. Stop.

5. Mating pool selection. Perform binary tournament selection with replacement on $P$ in order to fill the temporary mating pool $P^{\prime}$.

6. Variation. Apply recombination and mutation operators to the mating pool $P^{\prime}$ and add the resulting offspring to $P$. Go to Step 2.

The parameter $\delta>0$ is a specificity, representing the minimal value of the normalized function. Then, the preference based quality indicator $I_{p}$ can be defined as follows:

$$
I_{p}\left(x, x^{\prime}\right)=I_{\epsilon+}\left(x^{\prime}, x\right) / \sigma\left(f(x), z^{0}, \lambda, \rho, \delta\right)
$$

This quality indicator can now be used in (4) to set the fitness values of a population $P$. The resulting algorithm is referred to as PBEA.

In order to (approximately) give the same amplitude to each objective function, note that we replace each element $z_{i}$ of an objective vector $z$ with 
a normalized value by using the (approximated) ideal and nadir points:

$$
\frac{z_{i}-z_{i}^{\star}}{z_{i}^{n}-z_{i}^{\star}}
$$

Moreover, an external archive $A$ has been added to PBEA in order to store the current approximated efficient set. It is updated with new non-dominated solutions at each iteration of the algorithm.

\subsection{Outline of the Method}

The parallel multiple reference point approach proposed in this paper can be divided into two consecutive phases. The first one, called the preparation phase, is devoted to the design of several versions of a PBEA by $i$ ) estimating the bounds of the PF, ii) generating multiple reference points, and $i i i)$ designing a version of the solver for each reference point. The second phase is the running phase and consists of launching a PBEA version for every reference point in every processor, until a stopping condition is fulfilled. Details about the search method are summarized above.

\subsubsection{Preparation Phase}

The preparation phase is devoted to assign an equal number of $C P U_{\mathrm{s}}$ and reference point vectors by:

1. Getting a very rough common sense estimation of lower bounds and upper bounds for all objective functions. This estimation can either be given by the DM, or approximated automatically (see Subsection 3.1.1).

2. A possible stopping test for the latter algorithm can be stated as follows. First, determine the outer diameter (Chebychev norm of the distance between the current lower and upper bound) for the current efficient set approximation. Then, compare it with the former estimation of the outer diameter. If the difference between both values remains constant over a user-given number of iterations, stop.

3. Imagining a cutting plane through the area (of normalized ranges of objectives), and defining a uniform distribution of reference points in this area.

4. Assigning to every computer a PBEA version with a quality indicator function related to an achievement scalarization function defined by a reference point $z^{0}$ and a weighting vector $\lambda$. 


\subsubsection{Running Phase}

The running phase consists of performing a PBEA version in parallel until a stopping condition is verified. The output of each reference point solver is then merged to obtain the efficient set approximation. The overall algorithm is outlined next.

Parallel Multiple Reference Point Evolutionary Algorithm (PMRPEA).

1. Start with a user-given approximation of the objective ranges; use this approximation as an initial estimation of the bounds of the $\mathrm{PF}^{A}$ to determine the initial ideal and nadir point approximations, $z^{*^{A}}$ and $z^{n^{A}}$.

2. Set $d^{\star} \leftarrow$ outer diameter of these bounds, measured by $\left\|z^{n^{A}}-z^{*^{A}}\right\|$.

3. Set $\lambda=(1 / n, 1 / n, \ldots, 1 / n)$, where $n$ is the number of objective functions.

4. Generate $m$ initial reference points $z^{0(1)}, z^{0(2)}, \ldots, z^{0(m)}$ in a uniform way by using the (approximated) bounds of the PF.

5. Generate $m$ initial populations $P_{1}, P_{2}, \ldots, P_{m}$ of size $N$.

6 . For $i \leftarrow 1$ to $m$, perform in parallel (on $m$ processors):

$$
A_{i} \leftarrow \operatorname{PBEA}\left(P_{i}, \mathrm{G}, z^{0(i)}, \lambda, \rho\right)
$$

until a stopping condition is verified.

7. Return the non-dominated solutions of $A_{1} \cup A_{2} \cup \ldots \cup A_{m}$.

\section{Parallel Model and Implementation}

To design the algorithm proposed in the paper, two levels of parallelism may be used: the reference point level and the evolutionary algorithmic level. First, at the reference point level, using multiple reference points can naturally be implemented in a parallel algorithmic framework, since the resulting single-reference point solvers can all be launched independently. Moreover, we observed that multiple weighting coefficients vectors can also be used for the same reference point, what gives rise to a second level of parallelism. Next, at the evolutionary algorithmic level, three models can be distinguished (Melab et al., 2006). The first one is the Island model, which 
consists of deploying the same evolutionary algorithm on the islands with different parameters. In our case, let us remark that the reference points can be considered as the parameters. The second one, the parallel evaluation of the population consists of distributing the evaluation of the objective value(s) of solutions contained in the population between different "workers". The last one is the distributed evaluation of a single solution, where the evaluation of one solution is itself parallelized (when it is possible and interesting to do it). For the sake of simplicity, here we only focus our attention on the parallelism at the reference point level. To this end, we will implement the single reference point-based evolutionary algorithm as described in Subection 3.1.3. But, of course, any other reference point evolutionary algorithm can be used. For instance, a more advanced version of this evolutionary algorithm that would intrinsically involve parallelism can be considered instead of this simple model. The interested reader is referred to Melab et al. (2006) for more details about parallel and distributed metaheuristics.

In terms of implementation, ParadisEO (http://paradiseo.gforge. inria.fr) has been used. ParadisEO (Cahon et al., 2004) is a white-box object-oriented software framework devoted to the flexible design of metaheuristics. It is composed of four connected modules: ParadisEO-EO for population-based metaheuristics, ParadisEO-MO for single solution-based metaheuristics, ParadisEO-MOEO for multi-objective metaheuristics, and finally ParadisEO-PEO for parallel and distributed metaheuristics. Moreover, ParadisEO also includes tools for the design of hybrid and cooperative models. The single reference point evolutionary algorithm introduced in Subsection 3.1.3, PBEA, has been implemented using the ParadisEOMOEO (Liefooghe et al., 2009) module of ParadisEO. The ParadisEO-PEO module is based on the other modules of ParadisEO, so that PBEA instances can directly be used in the implementation of the parallel multiple reference point algorithm. Using ParadisEO-PEO, different types of parallel and distributed architectures may be used. In our case, the implementation of the parallel algorithm is based on the Message Passing Implementation (MPI) library. MPI is a parallel programming environment allowing a portable deployment on networks of heterogeneous workstations.

\section{A Case Study}

The FSP is one of the most well-known scheduling problems and has been widely studied in the literature. The majority of works devoted to this prob- 


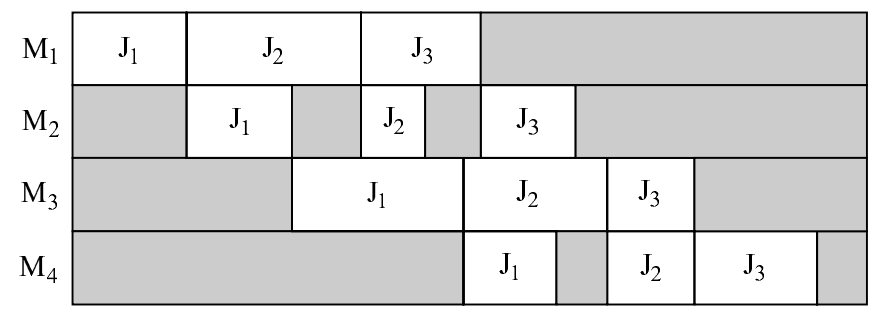

Figure 5: Example of a solution for a permutation FSP where 3 jobs $\left(J_{1}, J_{2}, J_{3}\right)$ have to be scheduled on 4 machines $\left(M_{1}, M_{2}, M_{3}, M_{4}\right)$.

lem considers it on a single-objective form and mainly aims at minimizing the makespan (i.e. the total completion time). However, many objective functions, varying according to the particularities of the tackled problem, may be considered and some multi-objective approaches have also been proposed. For a survey on this topic, see for instance Nagar et al. (1995); T'Kindt and Billaut (2002); Landa Silva et al. (2004); Minella et al. (2008).

Following the formulation of a multi-objective permutation FSP, this section presents some complexity issues and various works related to the problem under consideration.

\subsection{Problem Definition}

Solving the FSP consists of scheduling $N$ jobs $\left\{J_{1}, J_{2}, \ldots, J_{N}\right\}$ on $M$ machines $\left\{M_{1}, M_{2}, \ldots, M_{M}\right\}$. Machines are critical resources, i.e. one machine cannot process more than one job at a time. Each job $J_{i}$ is composed of $M$ consecutive tasks $\left\{t_{i 1}, t_{i 2}, \ldots, t_{i M}\right\}$, where $t_{i j}$ represents the $j^{\text {th }}$ task of the job $J_{i}$, requiring the machine $M_{j}$. A processing time $p_{i j}$ is associated to each task $t_{i j}$; and a due date $d_{i}$ is given to each job $J_{i}$ (the deadline of the job). In this study, we focus on the permutation FSP, where the operating sequences of the jobs are identical and unidirectional for every machine, as illustrated in Figure 5. Then, for a problem instance of $N$ jobs, there exists $N$ ! feasible solutions.

Many objective functions may be tackled while scheduling tasks on several machines (Nagar et al., 1995). The FSP that we consider here aims at minimizing the following ones: the makespan $\left(C_{\max }\right)$ and the total tardiness $(\bar{T})$. These objectives are among the most widely investigated in the literature (Nagar et al., 1995; T'Kindt and Billaut, 2002; Landa Silva et al., 2004; Minella et al., 2008). For each task $t_{i j}$ being scheduled at the time $s_{i j}$, 
they are computed as follows:

$$
\begin{gathered}
C_{\max }=\max _{i \in\{1, \ldots, N\}}\left\{s_{i M}+p_{i M}\right\} \\
\bar{T}=\sum_{i=1}^{N}\left\{\max \left\{0, s_{i M}+p_{i M}-d_{i}\right\}\right\}
\end{gathered}
$$

With respect to Graham et al. (1979), this problem can be denoted by $F /$ perm, $d_{i} /\left(C_{\max }, \bar{T}\right)$.

\subsection{Complexity Issues}

Even if it can be solved in polynomial time by using the Johnson's algorithm (Johnson, 1954) for two machines, the FSP of minimizing the makespan has been proven to be NP-hard for three or more machines (Lenstra et al., 1977). The objective of minimizing the total tardiness is already NP-hard for one machine (Du and Leung, 1990), what possibly explains the small number of studies dealing with minimizing the total tardiness in the case of $M$ machines (Kim, 1995). Hence, as minimizing the makespan and the total tardiness independently is already NP-hard, medium and large-size instances can generally not be solved exactly.

\subsection{Related Works}

The methods proposed in the literature for the resolution of multiple objective scheduling problems vary from exact methods, specific heuristics and metaheuristics. In their survey, Nagar et al. (1995) classify the scheduling problems according to different characteristics, including shop configuration (single or multiple machines) and objectives (two- or more-than-two objectives). The majority of FSP works on multiple machine has been restricted to the treatment of one objective at a time (generally the makespan or the sum of completion times). Moreover, most of multi-objective scheduling applications deal with two-machine and/or bi-objective problems, and concentrate on FSPs. T'Kindt and Billaut (2002) provide an overview on multiobjective scheduling for both researcher and industrial points of view. They provide models and a topology for single- and multi-objective scheduling problems, and describe some exact and heuristic algorithms to tackle them. Landa Silva et al. (2004) provided a survey about metaheuristics for solving multi-objective scheduling problems. It seems that the most commonly 


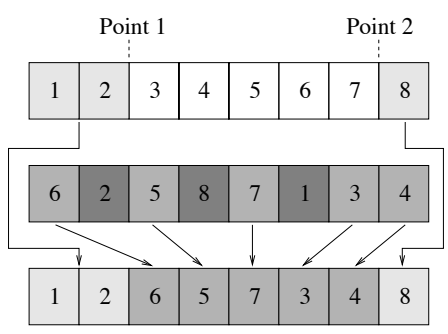

Figure 6: Insert mutation.

Figure 7: Two-point crossover.

used approaches are genetic algorithms and local searches, but also algorithms hybridizing both or hybridizing metaheuristics with exact methods. More recently, Minella et al. (2008) provided a review of the literature for multi-objective flowshop scheduling, together with a complete computational evaluation and comparison of a number of algorithms on some bi-objective variants of the problem.

\subsection{Problem-related Implementation Issues}

The problem-related components involved in the evolutionary algorithm, and used for the specific case of the FSP under consideration are the following ones:

- Individual representation: sequence of jobs scheduled in one machine. A solution of an instance with $N$ jobs and $M$ machines is represented by a permutation of size $N$.

- Initialization: Randomly generated solutions.

- Mutation: Insert mutation (Ishibuchi and Murata, 1998) (see Figure 6).

- Crossover: Two-point crossover (Ishibuchi and Murata, 1998) (see Figure 7).

\section{Computational Experiments}

In this section, computational experiments are performed on the parallel multiple reference point algorithm applied to the multi-objective FSP introduced in the previous section. The experimental protocol used to evaluate the quality of the algorithm is first described. Then, some results are given and discussed in order to extract some useful information about the proposed approach. 


\subsection{Experimental Protocol}

In order to quantify the effectiveness of the proposed approach, we compare its behavior to both NSGA-II (Deb et al., 2000) and IBEA (Zitzler and Künzli, 2004). The first one is often used as a reference to be compared with for MO. The latter is also investigated because the PMRPEA subprocedures are based on an IBEA-variant, so that the impact of the proposed parallelization scheme will be fairly measured at the implementation level.

\subsubsection{Characteristics of the Computer Network}

The experiments have been conducted on a cluster of 2 nodes, each one being composed of 2 Xeon 5060 CPUs (3.2 GHz, $2 \times 2 \mathrm{MB}, 4$ Gb RAM). Hence, a total number of 16 processors interconnected in a distributed computing environment were available.

\subsubsection{Benchmark Test Instances}

To evaluate the performance of the mechanisms introduced in this paper, we consider a set of benchmark test instances (Liefooghe et al., 2007). These benchmark test instances are available at the URL: http://www.lifl.fr/ riefooga/benchmarks/. They have been built from Taillard instances for the single-objective FSP (Taillard, 1993) and extended to the bi-objective case by adding a due date for every job. These due dates are fixed using a value randomly generated between $\bar{p} \times M$ and $\bar{p} \times(N+M-1)$, where $N$ stands for the number of jobs, $M$ for the number of machines and $\bar{p}$ for the average processing time for the instance under consideration. Thus, a due date roughly lies between the average completion date of the first scheduled job and the average completion date of the last scheduled job. During our experiments, we will consider a set of 8 instances involving between 20 and 100 jobs and between 5 and 20 machines. An instance denoted by $N \times M \times i$ represents the $i^{t h}$ instance composed of $N$ jobs and $M$ machines.

\subsubsection{Parameter Values Settings}

A preliminary experimental phase has been performed to determine the parameter values used during our experiments. They are summarized in Table 1. The stopping condition has been motivated by the fact that, after 5000 generations with no improvement, we can reasonably think that the evolutionary algorithm has reached its convergence. However, a nonimproving iteration is difficult to define while dealing with MO. Here, we state that an iteration is non-improving if there are no potential non-dominated 
Table 1: Parameter settings.

\begin{aligned} & \hline Parameter Value \\ & \hline Number of reference points 10 \\ & Population size 100 \\ & Max. number of iterations without improvement 5000 \\ & Maximum runtime 30 minutes \\ & Crossover rate 0.8 \\ & Mutation rate 1.0 \\ &$\kappa($ PBEA and IBEA) 0.05 \\ &$\delta(\mathrm{PBEA}) 10^{-2} \\ & \rho(\mathrm{PMRPEA}) 10^{-4} \\ &$\hline\end{aligned}

solutions that can be included into the archive. In addition, a maximum runtime of 30 minutes was allowed. Let us notice that the reference points have been specified as described in Subsection 3.1.2. The starting ideal and nadir point approximations were specified "manually"; that is, simulating initial information as if it was given by an experienced DM.

\subsubsection{Performance Assessment}

In the frame of $\mathrm{MO}$, the performance assessment of a number of algorithms in solving the same problem is a key issue. In this study, a set of 30 runs per instance is performed for each evolutionary algorithm. In order to evaluate the quality of the approximations for every instance we experimented, the protocol proposed by Knowles et al. (2006) was adopted. For a given instance, let $Z^{\text {all }}$ denotes the union of the outputs we obtained during all our experiments. Let us notice that this set probably contains both dominated and non-dominated points, as a given approximation may contain vectors dominating the ones of another approximation, and vice-versa. We first compute a reference set $\mathrm{PF}^{A}$ containing all the non-dominated points of $Z^{\text {all }}$ plus any other existing best know non-dominated set for the problem under consideration. Second, we define $z^{\max }=\left(z_{1}^{\max }, \ldots, z_{n}^{\max }\right)$, where $z_{k}^{\max }$ denotes the upper bound of the $k^{t h}$ objective for all the points contained in $Z^{\text {all }}$. In order to give a roughly equal range to the objective functions, values are normalized with respect to $z^{\max }$. Now, to measure the quality of an output set $A$ in comparison to $\mathrm{PF}^{A}$, we compute the difference between these two sets by using the unary hypervolume metric (Zitzler et al., 2003), $z^{\max }$ being the reference point. The hypervolume difference indicator $\left(\mathrm{I}_{H}^{-}\right)$com- 
putes the portion of the objective space that is weakly dominated by $\mathrm{PF}^{A}$ and not by $A$. The closer this measure is to 0 , the better is the approximation $A$. Furthermore, we also consider the additive $\epsilon$-indicator proposed in (Zitzler et al., 2003). The unary additive $\epsilon$-indicator $\left(\mathrm{I}_{\epsilon+}^{1}\right)$ gives the minimum factor by which an approximation $A$ can or has to be translated in the objective space to weakly dominate the reference set $Z_{N}^{\star}$. As a consequence, for each test instance, we obtain $30 \mathrm{I}_{H}^{-}$measures and $30 \mathrm{I}_{\epsilon+}$ measures, corresponding to the 30 simulation runs, per algorithm. Once all these values are computed, we first compute an average value per metric. Additionally, we perform a statistical analysis for a pairwise comparison of methods. To this end, we use the Wilcoxon signed rank test (Wilcoxon, 1945). Such a non-parametric statistical test is motivated by the fact that samples collected here correspond to matched samples. Indeed, for a given simulation run, the random seed numbers are identical for all the algorithms, so that the final indicator values can be taken as pairs. Details for this statistical testing procedure are given in (Knowles et al., 2006). Hence, for a given test instance, and with respect to the $p$-value and to the metric under consideration, this statistical test reveals that if the sample of approximation sets obtained by a given search method is significantly better than the one of another search method, or if there is no significant difference between both. Note that all the performance assessment procedures have been achieved using the performance assessment tool provided in PISA (Bleuler et al., 2003).

\subsection{Results and Discussion}

Results are summarized in Tables 2, 3, and 4. PMRPEA denotes the parallel multiple reference point evolutionary algorithm introduced in this paper. Let us notice that the running time was differently measured on the algorithms. The comparison between the algorithms is a little bit unfair because the computation load used for the parallel algorithm is, of course, higher than the existing ones. However, the latter have been designed and implemented in a sequential way by the original authors. According to the experimental protocol defined above, the first set of experiments revealed that PMRPEA results was significantly better than the ones of IBEA and NSGA-II on all instances with respect to both metrics. However, as it can be seen in Table 2, the average runtime of PMRPEA is always higher than the one of the other algorithms under investigation. That is the reason why we performed another set of experiments where the PMRPEA search process was stopped after a smaller runtime. The corresponding algorithm is de- 
Table 2: Comparison of the different algorithms with respect the average runtime, the average $\mathrm{I}_{H^{-}}^{-}$value and the average $\mathrm{I}_{\epsilon+}$-value.

\begin{tabular}{|c|c|c|c|c|}
\hline $\begin{array}{r}\text { benchmark } \\
\text { instance }\end{array}$ & & $\begin{array}{l}\text { average } \\
\text { runtime } \\
\text { (seconds) }\end{array}$ & $\begin{array}{r}\text { average } \\
\mathrm{I}_{H}^{-} \text {-value } \\
\left(\times 10^{-1}\right)\end{array}$ & $\begin{array}{r}\text { average } \\
\mathrm{I}_{\epsilon+} \text {-value } \\
\left(\times 10^{-1}\right)\end{array}$ \\
\hline \multirow[t]{4}{*}{$020 \times 05 \times 01$} & PMRPEA & 311.1 & 0.942 & 1.582 \\
\hline & PMRPEA-2 & 111.0 & 0.967 & 1.618 \\
\hline & IBEA & 111.5 & 1.905 & 2.046 \\
\hline & NSGA-II & 124.9 & 1.722 & 2.000 \\
\hline \multirow[t]{4}{*}{$020 \times 10 \times 01$} & PMRPEA & 345.0 & 0.212 & $\overline{0.492}$ \\
\hline & PMRPEA-2 & 147.0 & 0.229 & 0.508 \\
\hline & IBEA & 147.4 & 0.912 & 1.090 \\
\hline & NSGA-II & 182.6 & 0.760 & 1.035 \\
\hline \multirow[t]{4}{*}{$020 \times 20 \times 01$} & PMRPEA & 510.0 & 0.493 & 0.852 \\
\hline & PMRPEA-2 & 210.0 & 0.514 & 0.881 \\
\hline & IBEA & 210.7 & 1.900 & 2.446 \\
\hline & NSGA-II & 218.1 & 2.023 & 2.532 \\
\hline \multirow[t]{4}{*}{$050 \times 05 \times 01$} & PMRPEA & 517.1 & 0.740 & 0.738 \\
\hline & PMRPEA-2 & 254.0 & 0.750 & 0.745 \\
\hline & IBEA & 258.0 & 1.630 & 1.265 \\
\hline & NSGA-II & 254.2 & 1.522 & 1.231 \\
\hline \multirow[t]{4}{*}{$050 \times 10 \times 01$} & PMRPEA & 794.4 & 2.759 & 2.267 \\
\hline & PMRPEA-2 & 640.0 & 2.776 & 2.275 \\
\hline & IBEA & 640.1 & 3.398 & 2.855 \\
\hline & NSGA-II & 767.6 & 3.421 & 2.870 \\
\hline \multirow[t]{4}{*}{$050 \times 20 \times 01$} & PMRPEA & 1000.7 & 2.119 & 1.886 \\
\hline & PMRPEA-2 & 561.0 & 2.184 & 1.923 \\
\hline & IBEA & 561.0 & 3.558 & 2.995 \\
\hline & NSGA-II & 934.9 & 3.109 & 2.767 \\
\hline \multirow[t]{4}{*}{$100 \times 10 \times 01$} & PMRPEA & 1550.6 & 2.748 & 2.252 \\
\hline & PMRPEA-2 & 1060.0 & 2.817 & 2.303 \\
\hline & IBEA & 1060.5 & 4.643 & 4.028 \\
\hline & NSGA-II & 1260.3 & 3.889 & 3.367 \\
\hline \multirow[t]{4}{*}{$100 \times 20 \times 01$} & PMRPEA & 1740.5 & 2.666 & 2.280 \\
\hline & PMRPEA-2 & 1620.0 & 2.665 & 2.285 \\
\hline & IBEA & 1620.3 & 3.424 & 2.872 \\
\hline & NSGA-II & 1702.8 & 3.277 & 2.965 \\
\hline
\end{tabular}


Table 3: Wilcoxon rank test $p$-values with respect to the $\mathrm{I}_{H}^{-}$metric. Either the algorithm located at a specific row significantly outperforms the algorithm located at a specific column (represented by a gray or light-gray area for a $p$-value less or equal to 0.01 or to 0.05 , respectively), or there is no significant difference between both (represented by a white area).

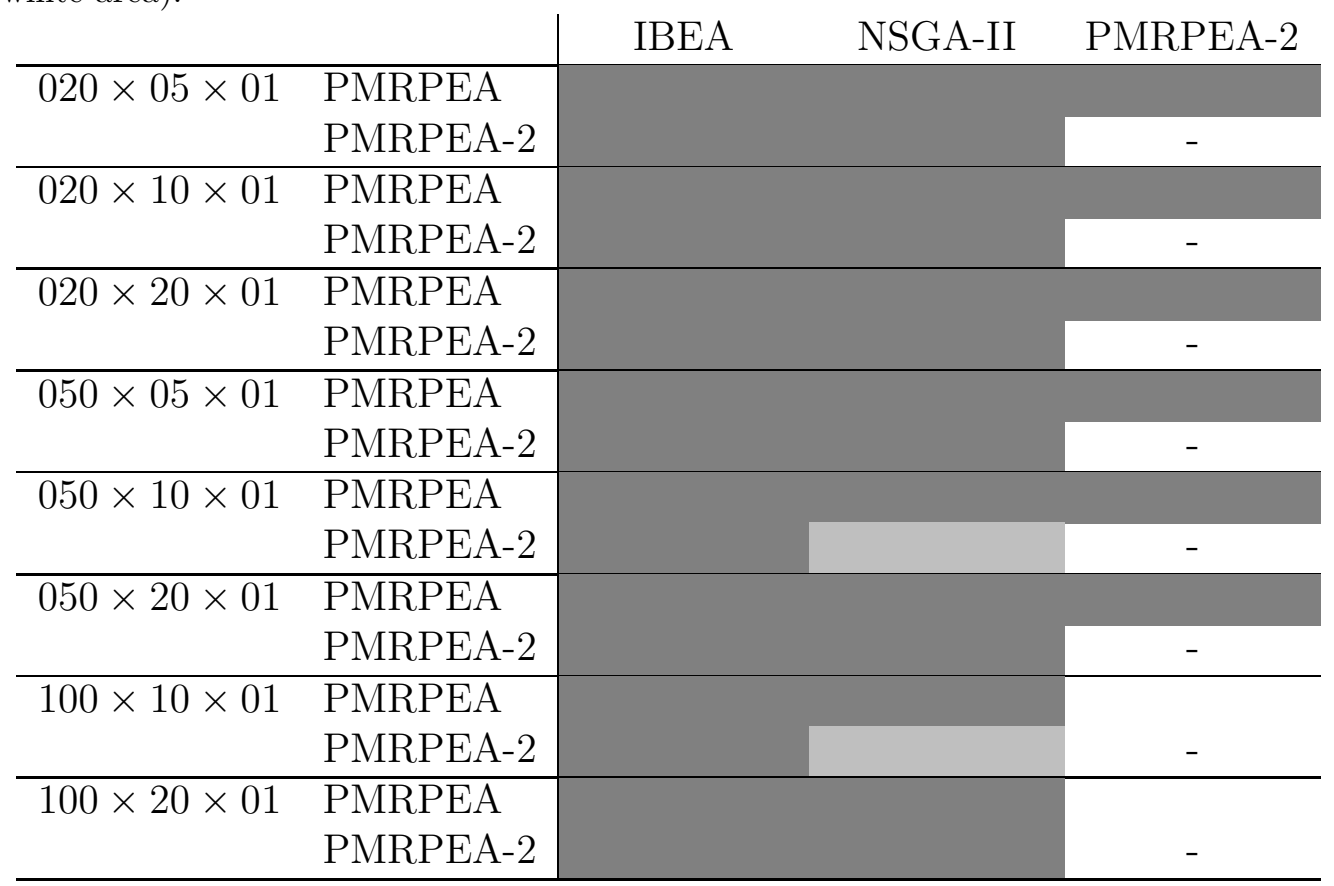

noted by PMRPEA-2. For a given benchmark test instance, the PMRPEA-2 maximum runtime was set as the minimum value between the average runtime of IBEA and the average runtime of NSGA-II, observed from previous experiments. Given that average runtime of our algorithm (PMRPEA) was higher than the remaining ones, it seems to us that the minimum average runtime of the other algorithms is a "natural" choice to verify that, with the same available CPU time, PMRPEA is still better than other algorithms. However, PMRPEA-2 also obtained significantly better $\mathrm{I}_{H^{-}}^{-}$and $\mathrm{I}_{\epsilon+}$-values than IBEA and NSGA-II on every instance we tested. Besides, PMRPEA-2 is predominantly outperformed by PMRPEA, except for large-size instances of 100 jobs, where the difference between both is not significant, see Tables 3 and 4 . 
Table 4: Wilcoxon rank test $p$-values with respect to the $\mathrm{I}_{\epsilon+}$ metric. Either the algorithm located at a specific row significantly outperforms the algorithm located at a specific column (represented by a gray or light-gray area for a $p$-value less or equal to 0.01 or to 0.05, respectively), or there is no significant difference between both (represented by a white area).

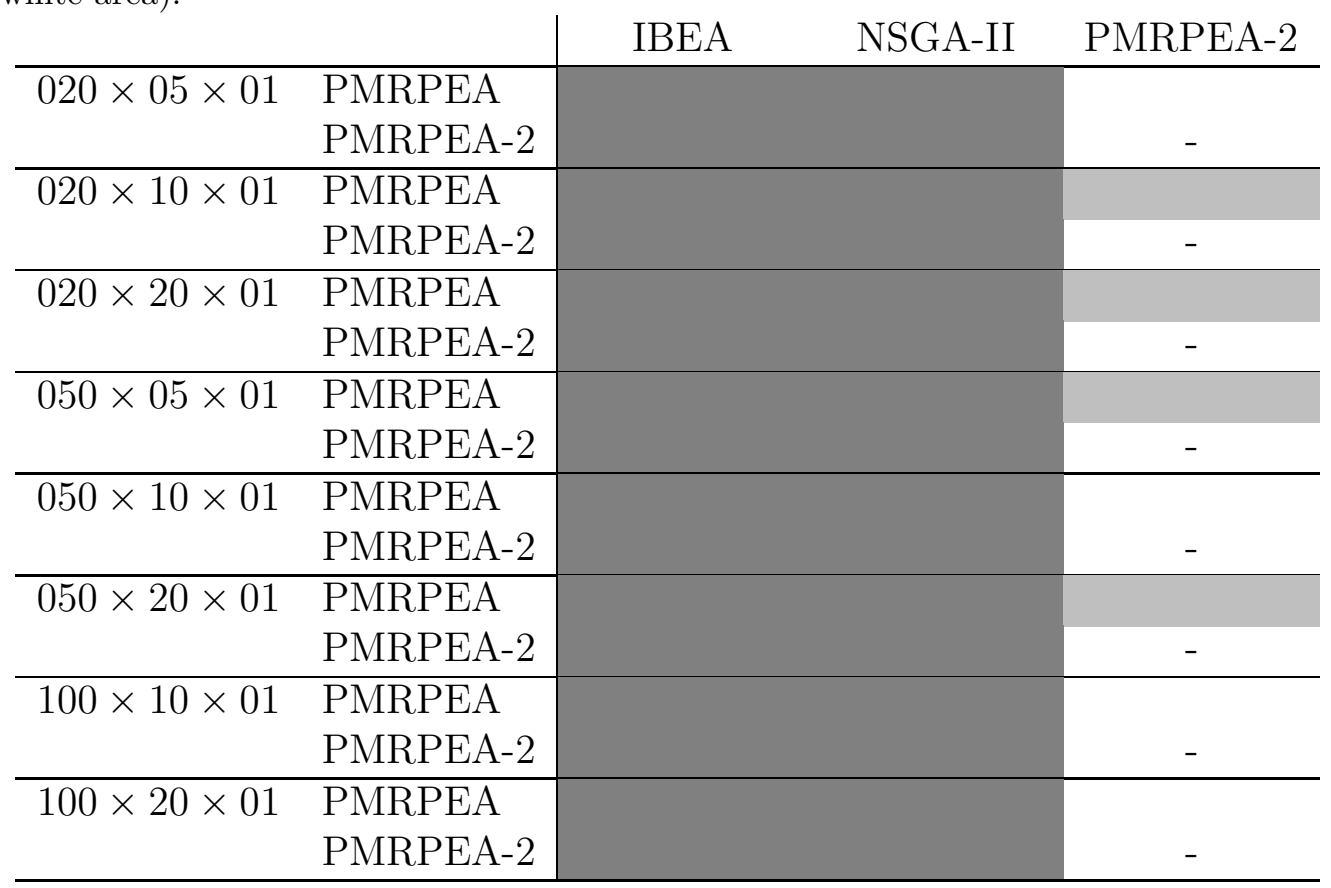

\section{Conclusion and Directions for Future Research}

This paper dealt with the design of a parallelization strategy to efficiently approximate the Pareto Frontier. Multiple reference points were used to uniformly divide the objective space into different areas. For each reference point, a set of approximate efficient solutions was found independently, so that the computation was performed in parallel. The proposed approach was applied to a real-world bi-objective combinatorial optimization problem which provided important results. In this parallel multiple reference point approach proposed for solving multi-objective optimization problems, the parallelization of evolutionary multi-objective optimization algorithms was combined with the use of a reference point approach. On the one hand, the problem can be formulated as follows: how to parallelize the resolution method by separating a number of regions in the objective space, then applying an evolutionary algorithm in each region, then combining the results? 
The clear motivation is to exploit the power of computer networks. On the other hand, the concept of reference points can be naturally used to separate regions of interest in the objective space, and then to parallelize the computations.

The outcomes of our tests show that the parallelization of evolutionary multi-objective optimization algorithms based on reference point methods gives very promising and statistically significantly better results than the comparable non-parallel evolutionary multi-objective algorithm. However, many further issues should be studied in future work. One of them is defining and analyzing statistically the speed-up factor due to parallelization. Another is analyzing diverse possible versions of parallelization, the impact of a larger number of objectives, etc. For example, it includes using different weighted vectors for the same reference point (Murata et al., 2001; Zhang and Li, 2007; Luque et al., 2009; Ruiz et al., 2009). Moreover, the issue of overlapping solutions resulting from the application of diverse reference points should be investigated in more detail, even if the results of experiments performed until now did not indicate that this issue is a major one. The concept of g-dominance can be used for such a purpose (Molina et al., 2009). An additional aspect for future research is to take into account the shape of the Pareto Frontier. A method for estimating this shape can be found in (Hernández-Díaz et al., 2007). Finally, interactive versions of the algorithms proposed in this paper are also possible, together with a question of possible dynamic modifications of reference points. Indeed, as it was previously pointed out, one of the fundamental aspects of reference point approaches, given from experiments, is the fact that the closer is a reference point to the Pareto Frontier, the faster it is to get a non-dominated outcome vector. Based on this observation, a dynamic update of reference points is suitable to reach non-dominated outcome vectors quicker. Figure 8 illustrates a way of updating a reference point. We can start with a first reference point, represented by a diamond $(\diamond)$ and get a first solution represented by a circle (o), that is the best found approximate solution with regards to the reference point and the achievement function under consideration. A simple rule for updating this reference point could be to use a linear convex combination to obtain a point in between the current reference point and the obtained solution. Proceeding in the same way, we can observe the path that leads to get a non-dominated outcome vector, represented by a bullet $(\bullet)$. Note that, if we use several linear combinations, we could spread the number of reference points and define another level of parallelization. 


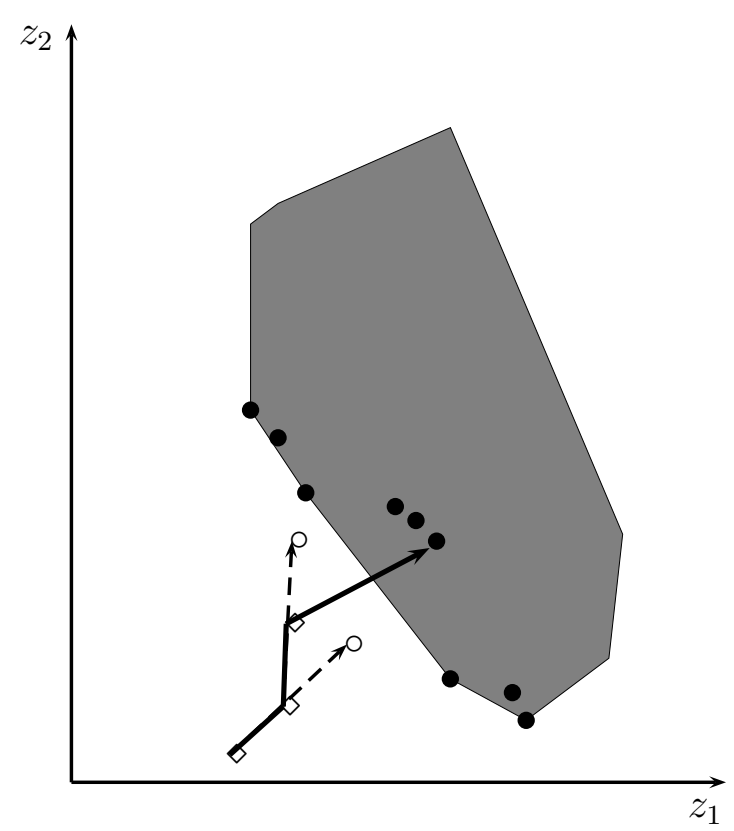

Figure 8: Dynamic reference points.

\section{Acknowledgments.}

The authors would like to acknowledge the three anonymous referees for their valuable comments and suggestions. José Rui Figueira acknowledges the COST Action Research Grant IC0602 on "Algorithmic Decision Theory".

\section{References}

Bleuler, S., Laumanns, M., Thiele, L., Zitzler, E., 2003. PISA - A platform and programming language independent interface for search algorithms. In: Fonseca, C. M., Fleming, P. J., Zitzler, E., Deb, K., Thiele, L. (Eds.), Conference on Evolutionary Multi-Criterion Optimization (EMO 2003). Vol. 2632 of Lecture Notes in Computer Science. Springer-Verlag, Faro, Portugal, pp. 494-508.

Cahon, S., Melab, N., Talbi, E.-G., 2004. ParadisEO: A framework for the reusable design of parallel and distributed metaheuristics. Journal of Heuristics 10 (3), 357-380.

Coello Coello, C. A., Van Veldhuizen, D. A., Lamont, G. B., 2002. Evolution- 
ary Algorithms for Solving Multi-Objective Problems. Kluwer Academic Publishers, Boston, MA, U.S.A.

Deb, K., 2001. Multi-Objective Optimization using Evolutionary Algorithms. John Wiley \& Sons, Chichester, U.K.

Deb, K., Agrawal, S., Pratab, A., Meyarivan, T., 2000. A fast elitist non-dominated sorting genetic algorithm for multi-objective optimization: NSGA-II. In: Proceedings of the 6th International Conference on Parallel Problem Solving from Nature (PPSN VI). Paris, France, pp. 849-858.

Deb, K., Chaudhuri, S., Miettinen, K., 2006. Towards estimating nadir objective vector using evolutionary approaches. In: Proceedings of the 8th Genetic and Evolutionary Computation Conference (GECCO 2006). Seattle, Washington, USA, pp. 643-650.

Du, J., Leung, J. Y.-T., 1990. Minimizing total tardiness on one machine is NP-hard. Mathematics of Operations Research 15, 483-495.

Graham, R. L., Lawler, E. L., Lenstra, J. K., Rinnooy Kan, A. H. G., 1979. Optimization and approximation in deterministic sequencing and scheduling: A survey. Annals of Discrete Mathematics 5, 287-326.

Hernández-Díaz, A. G., Santana-Quintero, L. V., Coello, C. A. C., Molina, J., 2007. Pareto-adaptive epsilon-dominance. Evolutionary Computation 15 (4), 493-517.

Ishibuchi, H., Murata, T., 1998. A multi-objective genetic local search algorithm and its application to flowshop scheduling. IEEE Transactions on Systems, Man and Cybernetics 28, 392-403.

Johnson, S. M., 1954. Optimal two- and three-stage production schedules with setup times included. Naval Research Logistics Quarterly 1, 61-68.

Kim, Y.-D., 1995. Minimizing total tardiness in permutation flowshops. European Journal of Operational Research 33, 541-551.

Knowles, J., Thiele, L., Zitzler, E., 2006. A tutorial on the performance assessment of stochastic multiobjective optimizers. Tech. rep., Computer Engineering and Networks Laboratory (TIK), ETH Zurich, Switzerland, (revised version). 
Landa Silva, J. D., Burke, E., Petrovic, S., 2004. An introduction to multiobjective metaheuristics for scheduling and timetabling. In: Gandibleux, X., Sevaux, M., Sörensen, K., T'kindt, V. (Eds.), Metaheuristics for Multiobjective Optimisation. Vol. 535 of Lecture Notes in Economics and Mathematical Systems. Springer-Verlag, Berlin, Germany, pp. 91-129.

Lenstra, J. K., Rinnooy Kan, A. H. G., Brucker, P., 1977. Complexity of machine scheduling problems. Annals of Discrete Mathematics 1, 343-362.

Liefooghe, A., Basseur, M., Jourdan, L., Talbi, E.-G., 2007. Combinatorial optimization of stochastic multi-objective problems: An application to the flow-shop scheduling problem. In: Obayashi, S., Deb, K., Poloni, C., Hiroyasu, T., Murata, T. (Eds.), Evolutionary Multi-criterion Optimization (EMO 2007). Vol. 4403 of Lecture Notes in Computer Science. SpringerVerlag, Matsushima, Japan, pp. 457-471.

Liefooghe, A., Jourdan, L., Talbi, E.-G., 2009. A unified model for evolutionary multiobjective optimization and its implementation in a general purpose software framework: ParadisEO-MOEO. Working Paper RR-6906, INRIA.

Luque, M., Miettinen, K., Eskelinen, P., Ruiz, F., 2009. Incorporating preference information in interactive reference point methods for multiobjective optmization. Omega - International Journal of Management Science 37 (2), 450-462.

Melab, N., Talbi, E.-G., Cahon, S., Alba, E., Luque, G., 2006. Parallel metaheuristics: Models and frameworks. In: Talbi, E.-G. (Ed.), Parallel Combinatorial Optimization. John Wiley \& Sons, Chichester, UK, Ch. 6, pp. 149-162.

Miettinen, K., 1999. Nonlinear Multiobjective Optimization. Vol. 12. Kluwer Academic Publishers, Boston, MA, U.S.A.

Minella, G., Ruiz, R., Ciavotta, M., 2008. A review and evaluation of multiobjective algorithms for the flowshop scheduling problem. INFORMS Journal on Computing 20 (3), 451-471.

Molina, J., Santana, L. V., Hernández-Díaz, A. G., Coello Coello, C. A., Caballero, R., 2009. g-dominance: Reference point based dominance for 
multiobjective metaheuristics. European Journal of Operational Research 167 (2), 685-692.

Murata, T., Ishibuchi, H., Gen, M., 2001. Specification of genetic search directions in cellular multi-objective genetic algorithms. In: Evolutionary Multi-criterion Optimization (EMO 2001). Vol. 1993 of Lecture Notes in Computer Science. Springer-Verlag, Berlin, Germany, pp. 82-95.

Nagar, A., Haddock, J., Heragu, S., 1995. Multiple and bicriteria scheduling: A literature survey. European Journal of Operational Research 81, 88-104.

Ruiz, F., Luque, M., Cabello, J. M., 2009. A classification of the weighting schemes in reference point procedures for multiobjective programming. Journal of Operational Research Society 60 (4), 544-553.

Steuer, R. E., 1986. Multiple Criteria Optimization: Theory, Computation, and Application. John Wiley \& Sons, Chichester, U.K.

Szczepański, M., Wierzbicki, A., 2003. Application of multiple criteria evolutionary algorithms to vector optimisation, decision support and reference point approaches. Journal of Telecommunications and Information Technology $3,16-33$.

Taillard, E. D., 1993. Benchmarks for basic scheduling problems. European Journal of Operational Research 64, 278-285.

Thiele, L., Miettinen, K., Korhonen, P. J., Molina, J., 2009. A preferencebased evolutionary algorithm for multi-objective optimization. Evolutionary Computation 17 (3), 411-436.

T'Kindt, V., Billaut, J.-C., 2002. Multicriteria Scheduling: Theory, Models and Algorithms. Springer-Verlag, Berlin, Germany.

Wierzbicki, A., 1980. The use of reference objectives in multiobjective optimization. In: Fandel, G., Gal, T. (Eds.), Multiple Objective Decision Making, Theory and Application. No. 177 in Lecture Notes in Economics and Mathematical Systems. Springer-Verlag, pp. 468-486.

Wierzbicki, A. P., Makowski, M., Wessels, J. (Eds.), 2000. Model-Based Decision Support Methodology with Environmental Applications. Kluwer Academic Publishers, Dordrecht. 
Wilcoxon, F., 1945. Individual comparisons by ranking methods. Biometrics 1, 80-83.

Zhang, Q., Li, H., 2007. MOEA/D: A multiobjective evolutionary algorithm based on decomposition. IEEE Trans. Evolutionary Computation 11 (6), $712-731$.

Zitzler, E., Künzli, S., 2004. Indicator-based selection in multiobjective search. In: Proceedings of the 8th International Conference on Parallel Problem Solving from Nature, Birmingham, U.K. (PPSN VIII). Vol. 3242 of Lecture Notes in Computer Science. Springer-Verlag, Berlin, Germany, pp. $832-842$.

Zitzler, E., Thiele, L., Laumanns, M., Foneseca, C. M., Grunert da Fonseca, V., 2003. Performance assessment of multiobjective optimizers: An analysis and review. IEEE Transactions on Evolutionary Computation 7 (2), $117-132$. 